\title{
Sistema de riego con movilidad a base de energía solar
}

\section{Irrigation system with mobility based on solar energy}

\author{
Dayaneth Arrocha ${ }^{l}$, Abel Camargo ${ }^{l}$, Jorge Petit ${ }^{1}$, Yineska Rosas ${ }^{1}$, Vladimir Villarreal ${ }^{*}$, Lilia Muñoz ${ }^{2}$ \\ ${ }^{1}$ Licenciatura en Ingeniería de Sistemas y Computación, Centro Regional De Chiriquí, Universidad Tecnológica de Panamá, \\ ${ }^{2}$ Facultad de Ingeniería de Sistemas Computacionales, Centro Regional de Chiriquí, Universidad Tecnológica de Panamá
}

Resumen Este proyecto trata de un robot que riega los cultivos mientras se moviliza con energía solar, que será almacenada en baterías, para que cuando el clima no le favorezca tenga energía para continuar trabajando. Además, tendrá la capacidad de detectar si hay personas en las parcelas, hacer un alto o cambiar la ruta y así poder evitar accidentes. El sistema también es capaz de detectar la temperatura cuando inicia el proceso de riego. Se ha decidido implementar la energía solar, ya que las máquinas utilizan petróleo que al quemarse generan grandes cantidades de desechos dañinos para el medio ambiente. Esto trae como consecuencia el cambio climático. la energía solar no contamina y es gratis, es por esto que tenemos que aprovechar esta fuente de energía renovable para capturarla y almacenarla. Aquí se transforman los fotones en energía eléctrica que usamos para darle vida a nuestro entorno y así no causaremos daños irreparables a nuestro medio ambiente.

Palabras clave Arduino, energía, motor, riego, sensores.

\begin{abstract}
This project is about a robot that irrigates crops while it is mobilized with solar energy, which will be stored in batteries, so that when the weather does not favor it, it has energy to continue working. In addition, it will have the ability to detect if there are people on the plots, stop or change the route so that accidents can be avoided. The system is also capable of detecting the temperature when the irrigation process starts. It has been decided to implement solar energy, since the machines use oil that, when burned, generates large amounts of waste that is harmful to the environment. This results in climate change. Solar energy does not pollute and it is free, that is why we have to take advantage of this renewable energy source to capture and store it. Here the photons are transformed into electrical energy that we use to give life to our environment and thus we will not cause irreparable damage to our environment.
\end{abstract}

Keywords Arduino, energy, engine, irrigation, sensors.

* Corresponding author: vladimir.villarreal@utp.ac.pa

\section{Introducción}

El uso del riego en la agricultura es una práctica muy antigua, desarrollada con la finalidad de proveer una cantidad adecuada de agua para el correcto desarrollo de los cultivos y permitir así la producción de alimentos en la época seca, en la cual no existen lluvias frecuentes [1]. Existen máquinas que ayudan a los agricultores a facilitar sus labores cotidianas, pero para esto necesitan ayuda de un personal que se encarga de manejar estos equipos y los mismos son a base de combustible el cual al quemarse se convierte en humo que es perjudicial para el medio ambiente.

El presente trabajo consiste en realizar un sistema programable con dos etapas, ya sea riego o fumigación, para un cultivo y se implementará tecnología abierta como es el caso del Arduino. La placa Arduino utiliza lógica digital permitirá que el riego o fumigación sea más eficiente generando una cosecha de mejor calidad y mayor producción.
También se utilizará un panel solar que se encargará de trasmitir energía a todo el sistema. Esta energía alimentará un circuito lógico que será el que da órdenes al motor o bomba eléctrica para llevar a cabo el funcionamiento.

\section{Antecedentes}

La entrada de los robots en la producción agrícola y ganadera choca muchas veces con el escepticismo de quienes creen que no podrán sustituir a los grandes tractores. Por ahora proporcionan más incrementos de productividad en las explotaciones más pequeñas o en cultivos específicos [2]. Cada día se están diseñando y construyendo diferentes tipos de robots que trabajen con total autonomía solar. Cada robot está equipado con células fotovoltaicas que le permiten, a la luz solar media, dirigir la electricidad recogida a sus motores y enviar la energía excedente recogida al litio [3]. Se está utilizando la tecnología para minimizar el impacto de la 
agricultura en el medio ambiente $\mathrm{y}$, al mismo tiempo, mejorar la calidad de vida [4]. Se realizan pruebas actualmente con los robots agrícolas, con el fin de mejorar los algoritmos para que puedan detectar con más precisión las malas hierbas [4].

Por su parte, la Powering Agriculture presentó el informe anual de 2018 que describe las actividades clave que impulsó la agricultura: un gran desafío energético para el desarrollo (PAEGC) que se llevó a cabo entre octubre de 2017 y septiembre de 2018 y que demuestran la importancia de utilizar la energía solar para la agricultura [5].

Según Best, expresó que los sistemas solares fotovoltaicos, por la flexibilidad de su aplicación, representan una oportunidad única para que el sector de la energía proporcione "paquetes" de servicios a las zonas rurales apartadas, por ejemplo, para los servicios de salud, educación, comunicaciones y luz eléctrica, así como para la agricultura y el suministro de agua [6].

Muchos han sido las soluciones que permite activar un sistema de riego a base de energía solar. El sistema se activa de forma fija, sin necesariamente estar en movimiento el sistema [7-10]. Nuestro proyecto además de utilizar la energía solar para activar el riego o fumigación también utiliza la energía para movilizar el dispositivo, que en nuestro caso es dispositivo con ruedas que carga y mueve todo el sistema. Esto facilita la reubicación del sistema de riego según donde se necesite.

\section{Materiales}

En el desarrollo de este proyecto se necesitaron algunos materiales, presentados a continuación (figura 1):

- Placa de Arduino Uno.

- Panel solar de 9V.

- Mini bomba sumergible 5V.

- Módulo de relé de alto nivel de 5V, es un interruptor que cierra o abre otros circuitos.

- Baterías de 9V recargables para el almacenamiento de la energía que envía el panel.

- Protoboard: es en el que se pueden insertar componentes electrónicos y cables para armar circuitos.

- Tubo de vinilo grado 5/16.

- Sensores DHT11 es el encargado de detectar la humedad y la temperatura.

- Sensor HC-SR04 se encarga de mostrar la distancia.

- Puente H sirve para el control de un motor de corriente directa.

- Robot Smart Car chassis kit

- Jumper Wires Ribbon Cables Kit.

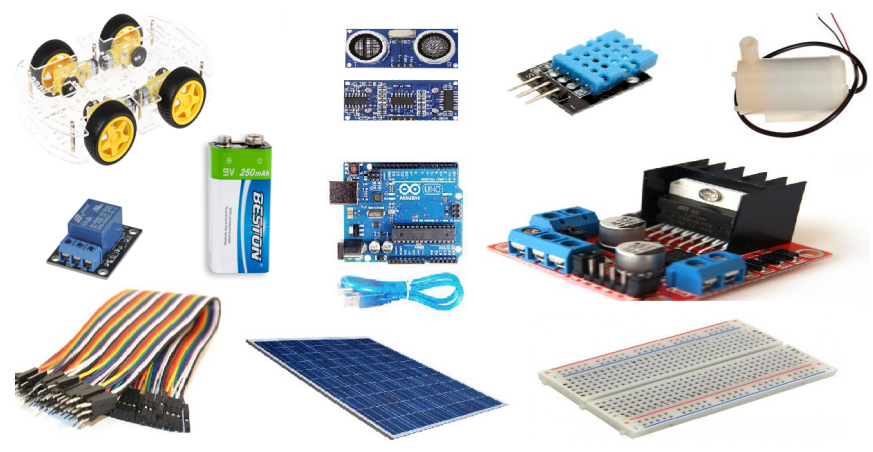

Figura 1. Materiales para el diseño del prototipo.

\section{Proceso de ensamblaje}

En este punto se presentará por medio de fases todas las realizaciones del proyecto:

\subsection{Fase 1}

Recolección de materiales que serán utilizados en la elaboración de dicho proyecto.

\subsection{Fase 2}

Se inicia soldando con estaño los diferentes cables a los motores que llevaran la energía al Robot Smart Car chassis kit para su funcionamiento, luego se le adaptaron las ruedas. Se realiza la prueba de los sensores DHT11 y HC-SR04, para saber si su función con el código implementado es la correcta o si se tienen que hacer modificaciones. Este proceso se puede observar en la figura 2.

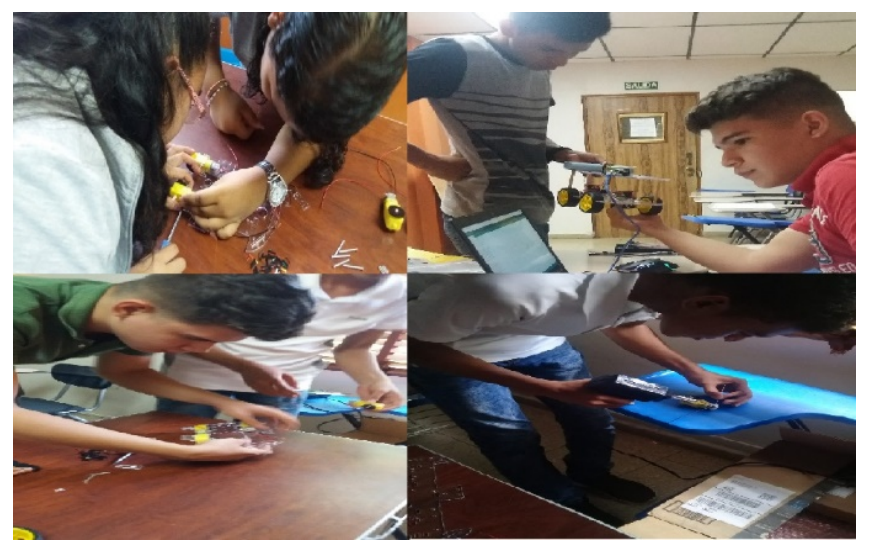

Figura 2. Pruebas iniciales de programación, ensamblaje y puesta en marcha.

\subsection{Fase 3}

Se unió el puente $\mathrm{H}$ al robot y se le colocaron los cables de los motores, luego se instaló el cableado que va del Arduino al puente $\mathrm{H}$, para así programar las ruedas para su funcionamiento, como se muestra en la figura 3.

Posteriormente se programó la bomba que se encargará de regar y fertilizar las parcelas. Dichas pruebas arrojaron 
resultados positivos, los cuales no permitieron avanzar a la siguiente fase.

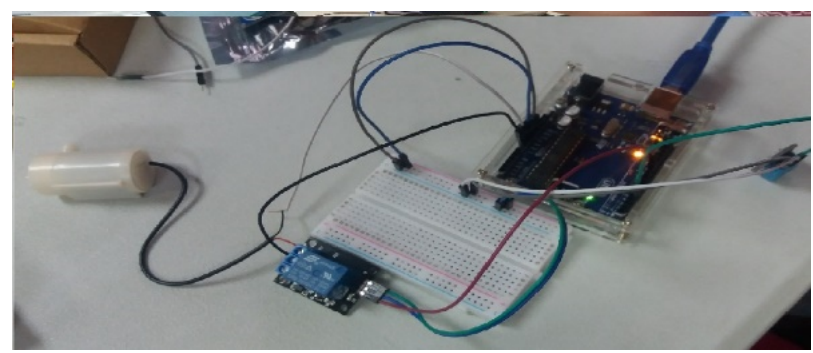

Figura 3. Comprobación de código en Arduino para arranque de la bomba y movimiento del carrito.

\subsection{Fase 4}

En esta última fase se unen todos los materiales utilizados en el chassis para probar su funcionamiento en conjunto (figura 4). Se hacen algunos cambios en la unión de códigos como pruebas tentativas para saber si la implementación está correcta.

\section{Funcionamiento del sistema}

En este espacio se muestra el funcionamiento del sistema implementando todos los materiales requeridos.

En la figura 5, el sistema espera la orden del usuario para comenzar su trabajo. El usuario presiona el botón de encendido para que haga su función. En este momento detectará el nivel de temperatura y humedad, si ambas están dentro del rango y la distancia que marque el sensor sea mayor que $15 \mathrm{~cm}$ se encenderán los motores que harán movilizar las ruedas y luego iniciar el riego. En el instante en el que el mismo detecte una persona u objeto en la parcela, detendrá el sistema de riego y apagará los motores de las ruedas para así evitar accidentes. Una vez concluido su ciclo el mismo regresará a su punto inicial.

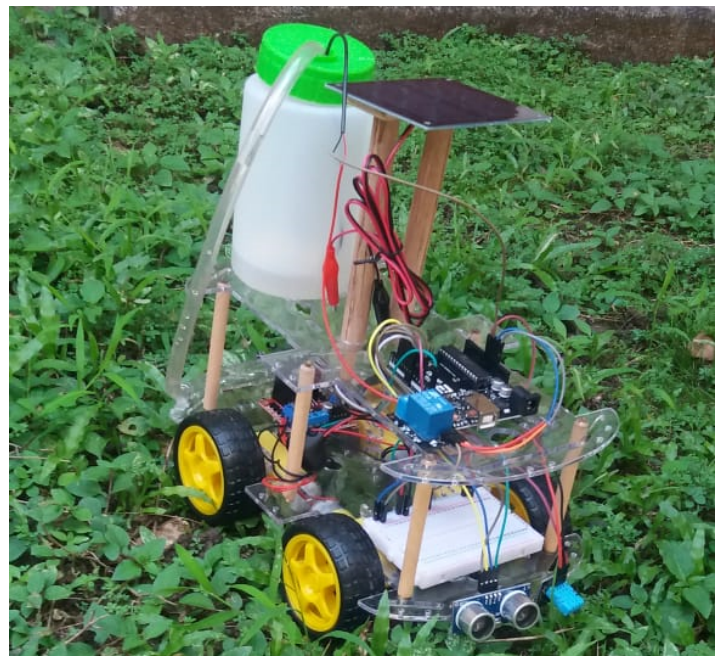

Figura 4. Unión de los materiales.

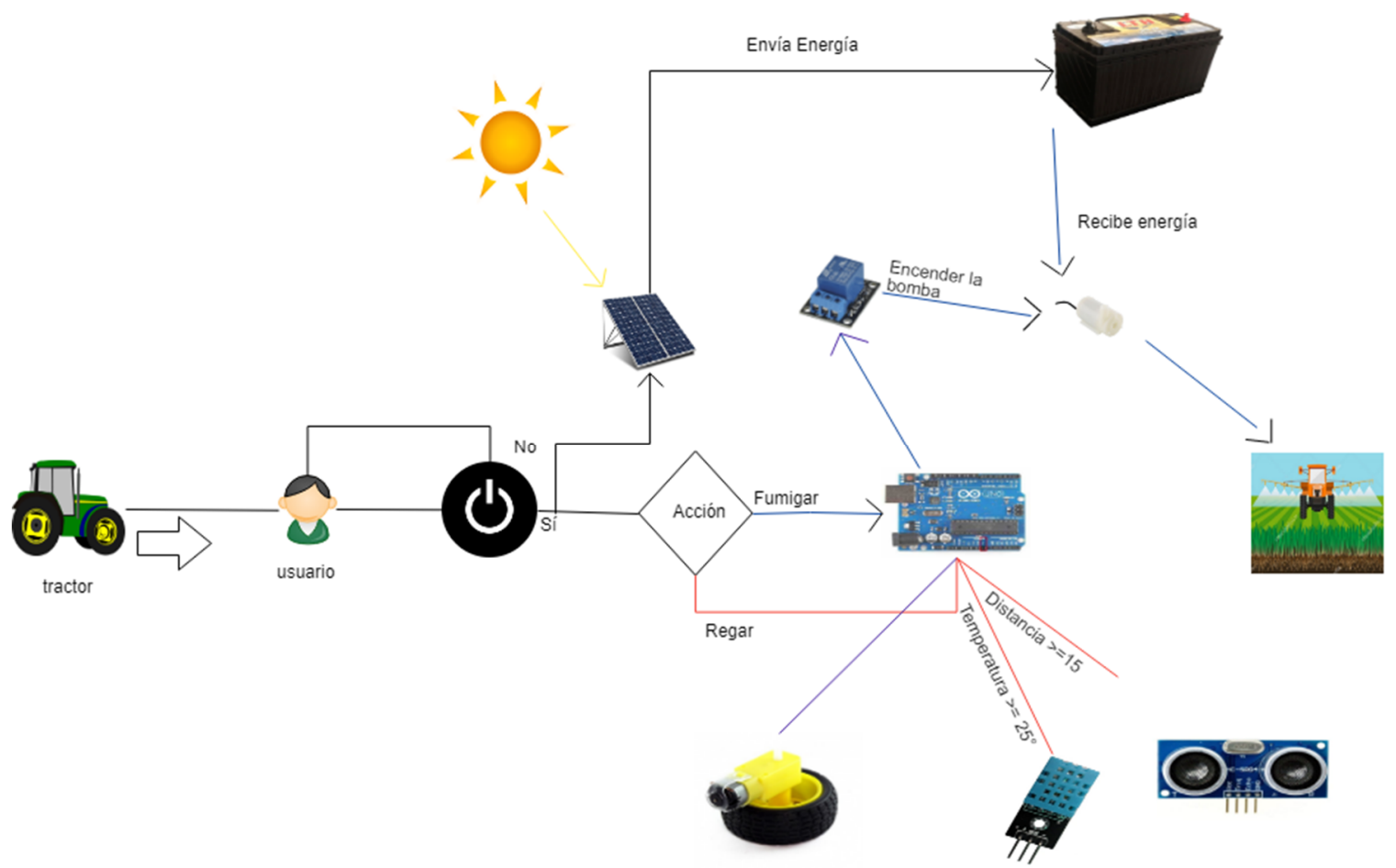

Figura 5. Diagrama Funcional. 


\section{Resultados}

Después de un largo periodo de pruebas realizadas entre sensores, mini bomba sumergible, Arduino y motores, se obtienen los siguientes resultados.

\begin{tabular}{|l|}
\hline Com5 \\
\hline Riega \\
Temperature $=24^{\circ} \mathrm{C}$, Distance $=173 \mathrm{~cm}$ \\
Riega \\
Temperature $=24^{\circ} \mathrm{C}$, Distance $=172 \mathrm{~cm}$ \\
Riega \\
Temperature $=24^{\circ} \mathrm{C}$, Distance $=172 \mathrm{~cm}$ \\
Riega \\
Temperature $=24^{\circ} \mathrm{C}$, Distance $=174 \mathrm{~cm}$ \\
Riega \\
Temperature $=24^{\circ} \mathrm{C}$, Distance $=172 \mathrm{~cm}$ \\
Riega \\
Temperature $=24^{\circ} \mathrm{C}$, Distance $=173 \mathrm{~cm}$ \\
Riega \\
Temperature $=24^{\circ} \mathrm{C}$, Distance $=172 \mathrm{~cm}$ \\
Riega \\
\hline Autoscroll $\square$ Mostrar marca temporal \\
\hline
\end{tabular}

Figura 6. Registro de la temperatura y distancia para regar.

En la figura 6 se puede apreciar la compilación de cómo funciona la mini bomba sumergible, en ella se ve que de acuerdo a la condición puesta en nuestro código ella hace la función de regar.

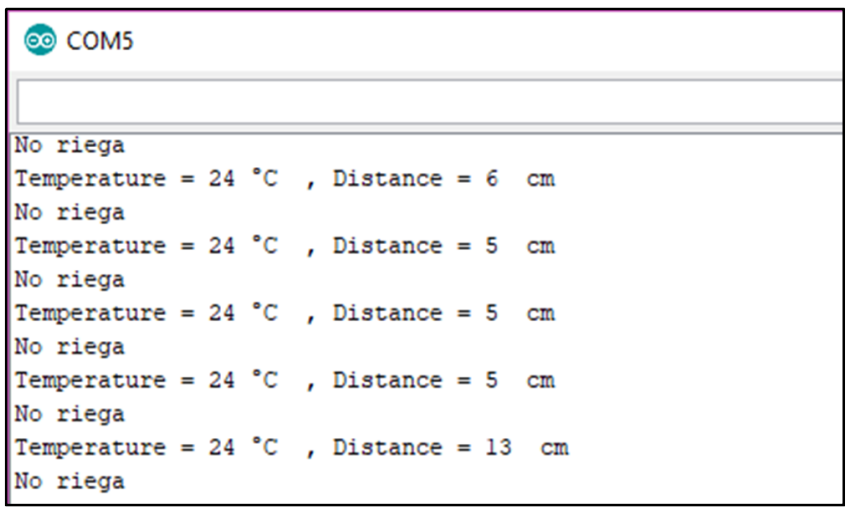

Figura 7. Registro de la temperatura y distancia para no regar.

Se aprecia en la figura 7 que como no cumple la condición dada, no regará.

El sistema de riego está listo para ser utilizado y brindar una ayuda a los agricultores o personas que necesiten implementarlo en sus negocios (figura 8).

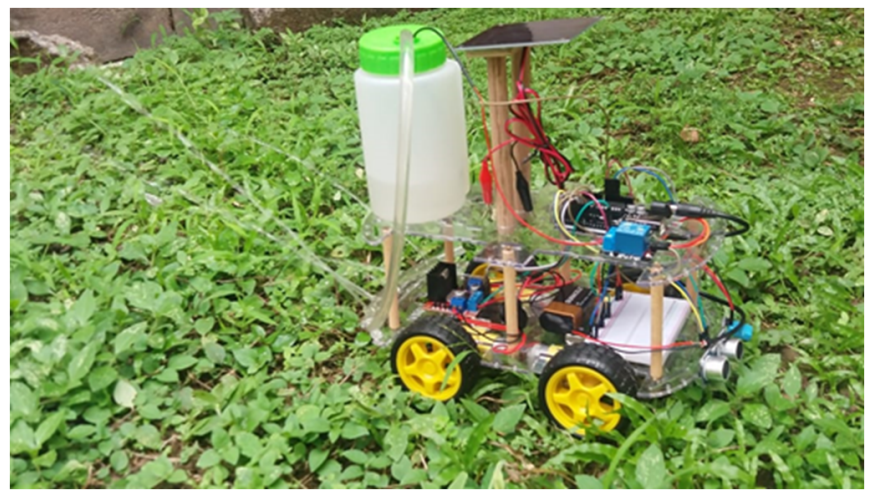

Figura 8. Sistema de riego.

\section{Conclusiones}

La generación de soluciones como estas, permiten solucionar el problema de riego controlado por dispositivos de bajo costo. Este sistema proporcionará ayuda para los agricultores, ya que representará un gran ahorro en cuanto a combustible, y mejorará la producción de los cultivos, además contribuirá con el medio ambiente, ya que en la actualidad se ve afectado con la contaminación del aire debido al tipo de combustibles que se utilizan en el campo.

Para ambientes de producción, el aprovechamiento de la energía solar resulta ser un elemento decisivo en cuanto a la optimización de recursos en los productores de nuestro pais.

Podemos controlar aspectos como temperatura, consumo de energía, distancia requerida para movilizar los equipos, entre otros aspectos o indicadores para mejorar la producción. No sólo en este tipo de proyectos sino en todas las fases de desarrollo e implemenatción de nuevas tecnologías.

El proyecto desarrollado puede ser implementado en huertos o cultivos controlados y que requieran un equipo de facil movilización entre las parcelas.

\section{AGRADECIMIENTOS}

Le damos las gracias al Profesor Vladimir Villareal por motivarnos a realizar este proyecto que nos ha ayudado a obtener conocimientos y complementarlos con los nuestros, para así llevar acabo el funcionamiento de nuestro robot.

\section{REFERENCIAS}

[1] S. Cruz et al., "Sistema de Riego." 2015. [Online]. Available:https://www.academia.edu/11172639/sistema_d e_riego

[2] B. Isabel, "Los robots, un nuevo paradigma en la agricultura - Horticultura," 2018. [Online]. Available: https://www.interempresas.net/Horticola/Articulos/20628 6-Los-robots-un-nuevo-paradigma-en-la-agricultura.html. [Accessed: 06-Jun-2019]

[3] AgriExpo, "Robot agrícola de corte / autónomo / de energía 
solar / vitícola - Vitirover." [Online]. Available: http://www.agriexpo.online/es/prod/vitirover/product177234-28632.html. [Accessed: 08-Jun-2019].

[4] Cinco Noticias, "EcoRobotix, el robot agrícola autónomo y ecológico." [Online]. Available: https://www.cinconoticias.com/ecorobotix-robot-agricolaautonomo-y-ecologico/. [Accessed: 22-Apr-2019].

[5] Powering Agriculture. (2019). Powering Agriculture Annual Report FY 2018. Feb. 26, 2019 [Accessed: 6-Feb2020]

[6] Best, G., Van Campen, B., \& Guidi, D. (2000). Energía solar fotovoltaica para la agricultura y desarrollo rural sostenibles.
[7] Sinha S., 2000; The solar photovoltaic programme in India - draft; 2000 and personal communication.

[8] Alfaro Rocha, J. A., Corbacho Torregroza, A. S., \& Prada Hernandez, J. E. (2018). Análisis del internet de las cosas (IOT) y su aplicabilidad en el control remoto del sistema de riego de la finca La Josefa ubicada en Macondo zona bananera del departamento del Magdalena.

[9] Bustamante, A., \& Juniors, E. (2019). Energía solar fotovoltaica para mejorar el sistema de riego del caserío La Victoria provincia de Utcubamba.

[10]Aqueveque Medina, E. J. (2009). Bombeo de Agua para Riego en Cerro Calán Utilizando Energía Solar Fotovoltaica. 